\title{
Psicooncología
}

ISSN: 1696-7240

\section{Adaptación de la Escala de Activación Conductual para la Depresión (BADS) en pacientes con cáncer}

\author{
Yesica María Villanueva Torres ${ }^{1 *}$; Samuel Jurado Cárdenas²; Armando Ramírez Ramírez ${ }^{3}$
}

Recibido: 30 de noviembre de 2019 / Aceptado: 24 de febrero de 2020.

Resumen: Objetivo: adaptar la Behavioral Activation for Depression Scale (BADS) y someterla a análisis factorial confirmatorio en una muestra de pacientes oncológicos. Método: Estudio instrumental en dos fases, en la fase 1 se tradujo y adaptó la escala original (BADS) al castellano según los estándares de la American Psychological Association ( $\mathrm{n}=101)$, en la fase 2 se aplicó la escala obtenida en la fase anterior y se sometió a análisis factorial confirmatorio $(\mathrm{n}=341)$. Resultados: el análisis factorial confirmatorio arrojó una escala formada por cuatro factores y 12 reactivos, con un ajuste: $\mathrm{X}^{2}=$ $1,24 \mathrm{gl}=48 \mathrm{p}=, 118 ; \mathrm{CFI}=, 98, \mathrm{TLI}=, 98, \mathrm{RMSEA}=, 027, \mathrm{NFI}=, 93, \mathrm{RFI}=, 91, \mathrm{IFI}=, 98, \mathrm{PNFI}=, 68$; tuvo una correlación negativa media con los constructos de ansiedad y depresión. Conclusiones: la adaptación de la Escala de Activación Conductual para la Depresión dirigida a pacientes oncológicos, tiene propiedades psicométricas aceptables lo que permite tener un instrumento adecuado para medir el nivel de activación conductual en esta población.

Palabras clave: Activación Conductual; depresión; cáncer; análisis factorial confirmatorio; validez.

\section{[en] Adaptation of the Behavioral Activation for Depression Scale (BADS) in cancer patients}

\begin{abstract}
Objective: To adapt the Behavioral Activation for Depression Scale (BADS) and submit it to confirmatory factor analysis in an oncological patients sample. Method: Instrumental study in two phases, phase 1 followed the American Psychological Association standards to translate and adapt the original scale (BADS) to Spanish ( $\mathrm{n}=101)$, in phase 2 we applied the scale obtained in the previous phase and submitted it to confirmatory factor analysis $(n=341)$. Results: the confirmatory factorial analysis showed a scale consisting of four factors and 12 items, with a fit: $\mathrm{X}^{2}=1.24 \mathrm{gl}=48 \mathrm{p}=.118$; CFI $=.98$, $\mathrm{TLI}=.98, \mathrm{RMSEA}=.27, \mathrm{NFI}=.93, \mathrm{RFI}=.91, \mathrm{IFI}=.98, \mathrm{PNFI}=.68$; had a medium negative correlation with anxiety and depression constructs. Conclusions: The adaptation of the Behavioral Activation Scale for Depression aimed at cancer patients has acceptable psychometric properties, which allows have an adequate instrument to measure the level of behavioral activation in this population.
\end{abstract}

Keywords: Behavioral Activation; depression; cancer; confirmatory factor analysis; validity.

1 Yesica Maria Villanueva Torres. Facultad de Psicología Universidad Nacional Autónoma de México. México E-mail: yesicavit@gmail.com

2 Samuel Jurado Cárdenas. Facultad de Psicología Universidad Nacional Autónoma de México. México E-mail: jurado@unam.mx

3 Armando Ramírez Ramírez. Hospital Juárez de México. México E-mail: armando7771@hotmail.com

* Dirección de correspondencia: Yesica Maria Villanueva Torres. Facultad de Psicología Universidad Nacional Autónoma de México. México. E-mail: yesicavit@gmail.com. 
Sumario: 1. Introducción 2. Método 2.1. Fase 1 2.2. Fase 2 3. Discusión y Conclusiones 4. Agradecimientos 5. Referencias bibliográficas

Cómo citar: Villanueva Torres YM, Jurado Cárdenas S, Ramírez Ramírez A. Adaptación de la Escala de Activación Conductual para la Depresión (BADS) en pacientes con cáncer. Psicooncología 2020; 17: 25-39. doi: 10.5209/psic.68239

\section{Introducción}

Además de deterioro fisiológico el cáncer y las secuelas de su tratamiento generan deterioro psicológico. De acuerdo con la National Comprehensive Cancer Netwok ${ }^{(1)}$, los pacientes pueden experimentar distress o malestar psicológico, el cual se extiende en un continuo que puede llegar hasta problemas incapacitantes, como depresión, ansiedad, pánico, aislamiento social, crisis existencial y espiritual; de éstos los más frecuentes son la ansiedad y la depresión ${ }^{(2-4)}$.

Una intervención incluida en guías de manejo de distress, ansiedad y depresión en pacientes oncológicos es la Terapia de Activación Conductual (AC) ${ }^{(5)}$.Si tomamos en cuenta las limitaciones en el comportamiento manifiesto, aumento de problemas y molestias diarias a menudo reportados por los pacientes con cáncer, la AC ofrece algunas ventajas en comparación con otras intervenciones ya que es menos complicada, utiliza estrategias de programación de actividades y reducción de la evitación y favorece su adaptación a la enfermedad ${ }^{(6,7)}$.

Se han realizado estudios con pacientes con cáncer en los cuales se utiliza la AC para tratar síntomas de depresión, en éstos se han utilizado registros de activación conductual y la Environmental Reward Observation Scale (EROS) para medir el éxito o fracaso de la intervención ${ }^{(7-13)}$. Sin embargo cuando se realizan intervenciones psicológicas basadas en evidencia, es necesario tener instrumentos de evaluación adecuados que midan las variables de interés. Si bien la EROS se desarrolló a partir del modelo de AC su objetivo es cuantificar el reforzamiento ambiental que recibe el sujeto $^{(14)}$, no medir el constructo de activación conductual. La Escala de Activación Conductual para la Depresión, BADS (por sus siglas en inglés Behavioral Activation for Depression Scale), proporciona una medida del constructo de activación, entendida como respuesta funcional alternativa a la evitación, permite saber cuándo y cómo el paciente empieza a realizar actividades y sus patrones de evitación cambian a lo largo del tratamiento, lo cual es esencial en los programas de Activación Conductual ${ }^{(15)}$.

La BADS tiene propiedades psicométricas adecuadas, está formada por un total de 25 reactivos divididos en cuatro factores: Activación, Evitación/Rumiación, Deterioro escolar/laboral y Deterioro social; las cuatro tienen un buen ajuste con los índices del análisis confirmatorio SB $\mathrm{X}^{2}(\mathrm{df}=271)=677,54, \mathrm{p}<0,01$; RMSEA $=0,070,90 \%$ intervalo de confianza $=0,063-0,077$; NNFI $=0,93$; CFI $=0,94$; varianza explicada $44 \%$, consistencia interna alta $(\alpha=0,87)$ y confiabilidad testretest aceptable $(\mathrm{r}=0,74)$. Tiene siete opciones de respuesta que van de 0 a $6^{(15)}$.

Se han realizado dos adaptaciones al castellano de la escala, las cuales tienen propiedades psicométricas aceptables, la primera adaptación se realizó para usarse con estudiantes y personal de una universidad española ${ }^{(16)}$ mientras que la segunda adaptación estuvo dirigida a adultos mayores mexicanos ${ }^{(17)}$. Sin embargo estas adaptaciones no estuvieron dirigidas a pacientes oncológicos y cada vez que una 
escala se usa en un contexto nuevo o con un grupo de personas diferentes es necesario estimar sus propiedades psicométricas ${ }^{(18)}$. Por lo tanto el objetivo del presente trabajo fue adaptar la BADS y someter la escala a análisis factorial confirmatorio en una muestra de pacientes oncológicos mexicanos.

\section{Método}

Estudio instrumental en dos fases, ${ }^{(19)}$ el cual fue aprobado por los Comités de Investigación, Ética en Investigación y Bioseguridad del Hospital Juárez de México.

\subsection{Fase 1}

Esta fase tuvo como objetivos: traducir al español la BADS, someter la adaptación a evaluación de jueces expertos y aplicar el instrumento a una muestra mínima de 50 participantes para identificar dificultades para entender instrucciones, formato y reactivos problemáticos.

\section{Participantes}

Muestra intencional por cuota ${ }^{(20)}$ conformada por 101 pacientes con diagnóstico oncológico, 79 mujeres y 22 hombres con edad entre 20 y 84 años (Media $=51,73$, $\mathrm{DT}=12,80$ ), con puntaje igual o menor a 2 en el índice Eastern Cooperative Oncology Group (ECOG), sin retraso mental o deterioro cognitivo y que aceptaron participar en el estudio.

\section{Instrumentos}

- Behavioral Activation for Depression Scale (BADS) Escala desarrollada por Kanter et al. ${ }^{(15)}$ cuenta con propiedades psicométricas aceptables.

- Toxicity and response criteria of the Eastern Cooperative Oncology Group $(E C O G)^{(21)}$ Es una escala que mide la repercusión funcional de la enfermedad oncológica en el paciente como criterio de progresión, puntúa de 0 a 5 .

- Hoja de datos sociodemográficos: Formato con el cual se recolectaron datos.

\section{Procedimiento}

Se siguieron las fases propuestas por Carretero-Dios y Pérez ${ }^{(22,23)}$ para construir y adaptar instrumentos que tienen como objetivo la evaluación de constructos relacionados con la Psicología Clínica y de la Salud, estas fases se desarrollaron a partir de estándares internacionales.

Se realizó una traducción inversa del instrumento ${ }^{(22,23)}$, es decir la escala desarrollada por Kanter et al..$^{(15)}$ se tradujo del inglés al castellano, posteriormente se re-tradujo al inglés; esta versión se comparó con la escala original y los reactivos fueron equivalentes. La versión traducida al castellano del BADS fue evaluada por jueces expertos y se llegó a un acuerdo inter-jueces sobre la traducción de los reactivos.

La versión traducida al castellano de la BADS (versión A) conformada por 25 reactivos se aplicó a 51 pacientes de forma individual en la sala de espera del 
servicio de oncología. El investigador explicó a los participantes el objetivo, que su participación era voluntaria y podían retirase del estudio en el momento que desearan sin tener consecuencias en la atención que recibían en el hospital. A los pacientes que lo solicitaron, se les proporcionó información sobre a qué área del hospital podían acudir para solicitar atención psicológica, se les entregó el aviso de privacidad del hospital y consentimiento informado. Posteriormente se realizaron las modificaciones necesarias y se obtuvo una segunda versión de la escala al castellano BADS (versión B) la cual se aplicó a 50 pacientes, de acuerdo al procedimiento señalado.

\section{Resultados de la fase 1}

A pesar de que la escala BADS (versión A) al castellano conformada por 25 reactivos tuvo una consistencia interna adecuada $(\mathrm{alfa}=0,86)$, valores de asimetría y curtosis menores a 2,0 en todos los reactivos; el $76 \%$ de los participantes reportaron que el reactivo 15 "Frecuentemente pasé tiempo pensando en mi pasado, personas que me han lastimado, errores que he cometido y otras cosas malas en mi historia" era confuso, por lo que fue modificado en 3 reactivos diferentes: "Pasé tiempo pensando en mi pasado"; "Pasé tiempo pensando en personas que me han lastimado" y "Pasé tiempo pensando en errores que he cometido y otras cosas malas en mi historia". Después de la evaluación cualitativa de ítems ${ }^{(22,23)}$ se decidió mantener las siete opciones de respuesta de la escala original y modificar el formato de las opciones, como se observa en la figura 1. Una vez realizadas estas modificaciones se obtuvo una segunda versión de la escala al castellano BADS (versión B) conformada por 27 reactivos.

Figura 1. Formato de opciones de respuesta

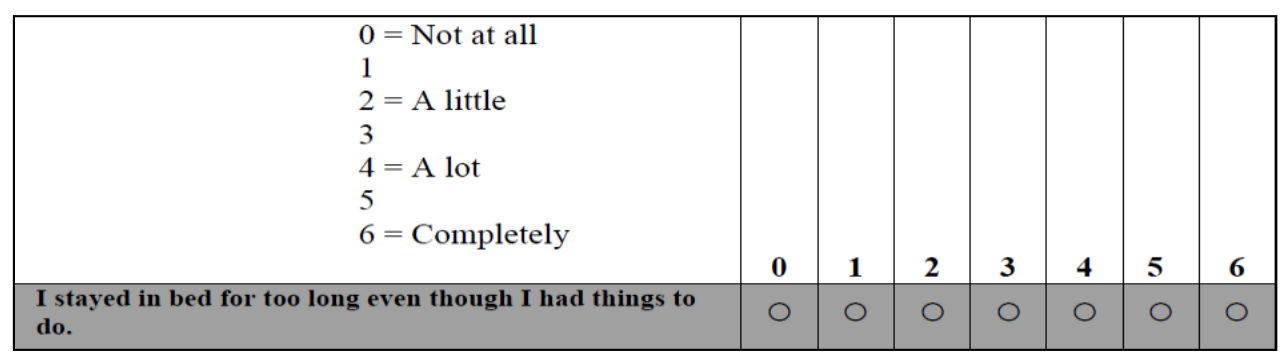

Opciones de respuesta de la escala original

\begin{tabular}{|c|c|c|c|c|c|c|c|}
\hline & $\begin{array}{c}\text { Nunca } \\
0\end{array}$ & $\begin{array}{c}\text { Casi } \\
\text { nunca } \\
1\end{array}$ & $\begin{array}{c}\text { Rara vez } \\
2\end{array}$ & $\begin{array}{c}\text { A veces } \\
3\end{array}$ & $\begin{array}{c}\text { Con } \\
\text { frecuencia } \\
4\end{array}$ & $\begin{array}{l}\text { Casi } \\
\text { siempre } \\
5\end{array}$ & $\begin{array}{c}\text { Siempre } \\
6\end{array}$ \\
\hline $\begin{array}{l}\text { Traté de no pensar en ciertas } \\
\text { cosas. }\end{array}$ & 0 & & & & & & \\
\hline
\end{tabular}

Opciones de respuesta de la adaptación al castellano 
Esta segunda versión al castellano BADS (versión B) con 27 reactivos tuvo un alfa de Cronbach de 0,84 , asimetría y curtosis menor a 2,0 en todos los reactivos (tabla 1) y las respuestas se distribuyeron en todas las opciones de la escala, ninguna opción tuvo más del $50 \%$ de las respuestas. Ningún participante refirió que la escala fuera confusa.

Tabla 1. Asimetría y curtosis por reactivo

\begin{tabular}{|c|c|c|}
\hline Reactivo & Asimetría & Curtosis \\
\hline 1 & $-1,275$ & ,361 \\
\hline 2 & $-1,141$ &,- 120 \\
\hline 3 &,- 788 &,- 633 \\
\hline 4 &,- 362 & $-1,264$ \\
\hline 5 &,- 791 &,- 616 \\
\hline 6 &,- 347 & $-1,276$ \\
\hline 7 &,- 739 &,- 531 \\
\hline 8 &,- 140 & $-1,486$ \\
\hline 9 & ,888 &,- 228 \\
\hline 10 & ,898 &,- 076 \\
\hline 11 & $-1,005$ &,- 177 \\
\hline 12 & $-1,073$ &,- 017 \\
\hline 13 & ,083 & $-1,472$ \\
\hline 14 &,- 613 & $-1,030$ \\
\hline 15 &,- 010 & $-1,499$ \\
\hline 16 &,- 423 & $-1,302$ \\
\hline 17 &,- 019 & $-1,419$ \\
\hline 18 &,- 704 &,- 841 \\
\hline 19 &,- 926 &,- 507 \\
\hline 20 &,- 852 &,- 858 \\
\hline 21 & $-1,289$ &, 530 \\
\hline 22 & $-1,145$ &,- 158 \\
\hline 23 &,- 800 &,- 995 \\
\hline 24 &,- 068 & $-1,640$ \\
\hline 25 &,- 523 &,- 999 \\
\hline 26 &,- 340 & $-1,364$ \\
\hline 27 &,- 736 & $-1,052$ \\
\hline
\end{tabular}




\subsection{Fase 2}

Esta fase tuvo como objetivo determinar las propiedades psicométricas de la adaptación al castellano de la BADS (versión B).

\section{Participantes}

Muestra intencional por cuota $^{(24)}$ conformada por 341 pacientes con diagnóstico oncológico, 290 mujeres y 51 hombres, con edad entre 20 y 85 años (Media= 51,70, $\mathrm{DT}=13,34)$, puntaje igual o menor a 2 en el ECOG, sin retraso mental o deterioro cognitivo, que aceptaron participar en el estudio, la descripción de los participantes se muestra en la tabla 2.

Tabla 2. Descripción de los participantes

\begin{tabular}{|c|c|c|c|c|c|}
\hline & $\mathbf{f}$ & $\%$ & & $\mathbf{f}$ & $\%$ \\
\hline Ocupación & & & Tipo de cáncer & & \\
\hline Ama de casa & 199 & 58,4 & Mama & 134 & 39,3 \\
\hline Comerciante & 34 & 10 & $\mathrm{Cacu}$ & 51 & 15 \\
\hline Desempleado/a & 16 & 4,7 & Ovario & 33 & 9,7 \\
\hline Oficio & 35 & 10,3 & Otro* & 20 & 5,9 \\
\hline Empleada doméstica & 11 & 3,2 & Colon & 19 & 5,6 \\
\hline Empleado/a & 21 & 6,2 & Endometrio & 16 & 4,7 \\
\hline Técnico o profesionista & 10 & 2,9 & Próstata & 13 & 3,8 \\
\hline Jubilado o pensionado & 5 & 1,5 & Testículo & 10 & 2,9 \\
\hline Estudiante o pasante & 5 & 1,5 & Estómago & 10 & 2,9 \\
\hline Otro & 5 & 1,5 & Melanoma & 7 & 2,1 \\
\hline Escolaridad & & & Riñon & 5 & 1,5 \\
\hline Sin escolaridad & 26 & 7,6 & Linfoma & 5 & 1,5 \\
\hline Primaria & 96 & 28,2 & Recto & 5 & 1,5 \\
\hline Secundaria & 97 & 28,4 & LNH & 5 & 1,5 \\
\hline Preparatoria & 84 & 24,6 & Tiroides & 4 & 1,2 \\
\hline Licenciatura & 37 & 10,9 & Sarcoma & 4 & 1,2 \\
\hline Posgrado & 1 & 0.3 & & & \\
\hline
\end{tabular}

\section{Instrumentos}

- Escala de Activación Conductual para la Depresión (BADS) (Versión B). Adaptación al castellano formada por 27 reactivos, desarrollada en la fase 1. 
- Toxicity and response criteria of the Eastern Cooperative Oncology Group $(E C O G)^{(21)}$. Es una escala que mide la repercusión funcional de la enfermedad oncológica en el paciente como criterio de progresión, puntúa de 0 a 5 .

- Escala de Ansiedad del Inventario Mexicano de Ansiedad, Depresión e Ideación Suicida $(I M A D I S)^{(25)}$. Mide la presencia de ansiedad en tres aspectos: cognitiva, conductual y fisiológica. Tiene propiedades psicométricas adecuadas alfa de Cronbach 0,96, adecuación de muestreo para ansiedad 0,96, gl 1711, p $<0$; CFI: 0,95, TLI: 0,94, RMSEA: 0,04, NFI: 0,93, RFI: 0,92, IFI: 0,95, PNFI: 0,82.

- Escala de Depresión del Inventario Mexicano de Ansiedad, Depresión e Ideación Suicida (IMADIS)(25). Considera: sesgos cognitivos, modos de procesamiento y estrategias de afrontamiento Posee propiedades psicométricas adecuadas alfa de Cronbach $=0,95$, adecuación de muestreo 0,96, gl 1081, $\mathrm{p}<0,0001$; CFI: 0,94, TLI: 0,93, RMSEA: 0,054, NFI: 0,91, RFI: 0,90, IFI: 0,94, PNFI: 0,84 .

- Versión Mexicana del Inventario de Ansiedad de Beck(26). Tiene una alta consistencia interna (alfa: 0,83 ), confiabilidad Test-Retest ( $\mathrm{r}=0,75)$, índice de correlación moderado positivo y $\mathrm{p}<0,05$ con el Inventario de Ansiedad Rasgo Estado (IDARE) y una estructura factorial de cuatro factores principales congruente con la referida por otros autores con la versión original.

- Versión Mexicana del Inventario de Depresión de Beck ${ }^{(27)}$. Tiene una alta consistencia interna (alfa: 0,87), una estructura factorial de tres factores congruente con la versión original, índice de correlación $r=0,70, p<0,0001$ con la Escala de Zung.

- Hoja de datos sociodemográficos. Formato con el cual se recolectaron los datos.

\section{Procedimiento}

La batería de pruebas se aplicó de forma individual en la sala de espera del servicio de oncología de acuerdo al procedimiento señalado en la fase uno, si deseaban saber su resultado en los instrumento podían solicitarlo al investigador. A los pacientes que presentaron niveles altos de ansiedad y/o depresión, se les proporcionó información sobre a qué área del hospital podían acudir para solicitar atención psicológica. Además de la hoja de datos sociodemográficos y la BADS, a 171 de los participantes se les entregó también el inventario de depresión de Beck y la escala de depresión del IMADIS; mientras que a 170 se les entregó el inventario de ansiedad de Beck y la escala de ansiedad del IMADIS. Esto con el objetivo de establecer la validez de constructo de la escala a través de la divergencia con otros constructos como ansiedad y depresión ${ }^{(22,23)}$; se planteó la hipótesis de que la BADS tendría una correlación negativa considerable con las escalas de ansiedad y una correlación negativa fuerte con las escalas de depresión.

\section{Resultados de la fase 2}

La distribución de frecuencias para cada reactivo mostró que las respuestas se distribuyen en todas las opciones de la escala. Los valores de asimetría y curtosis resultaron adecuados para todos los reactivos (menores a 2,0). El coeficiente de confiabilidad alfa de Cronbach de la escala resultó aceptable alfa $=0,83$, todos 
los reactivos correlacionaron positiva y significativamente con el puntaje total. La capacidad discriminativa de los reactivos se analizó a través de la prueba $t$ de Student, aquellos reactivos que no discriminaban $(p>0,05)$ se muestran en la tabla 3 .

Tabla 3. Asimetría, curtosis, capacidad discriminativa y confiabilidad por reactivo

\begin{tabular}{|c|c|c|c|c|c|c|c|}
\hline Reactivo & Asimetría & Curtosis & $\mathrm{t}$ & Gl & $\mathrm{P}$ & $\begin{array}{l}\text { Correlación } \\
\text { elemento-total } \\
\text { corregida }\end{array}$ & $\begin{array}{c}\text { Alfa de } \\
\text { Cronbach si } \\
\text { se elimina el } \\
\text { reactivo }\end{array}$ \\
\hline 1 & $-1,005$ & ,023 & $-2,944$ & 168 & 0,004 & 0,401 & 0,829 \\
\hline 2 &,- 513 &,- 787 & $-4,485$ & 168 &, 000 & 0,458 & 0,827 \\
\hline 3 &,- 709 &,- 779 & $-1,82$ & 168 & $0,071 *$ & 0,371 & 0,83 \\
\hline 4 &,- 410 & $-1,066$ & $-1,182$ & 168 & $0,239 *$ & 0,274 & 0,833 \\
\hline 5 &,- 804 &,- 380 & $-1,108$ & 168 & 0,270 * & 0,329 & 0,831 \\
\hline 6 &,- 492 &,- 806 & $-2,285$ & 168 & 0,024 & 0,33 & 0,831 \\
\hline 7 &,- 470 &,- 970 & $-3,129$ & 168 & 0,002 & 0,412 & 0,828 \\
\hline 8 &,- 265 & $-1,321$ & $-3,087$ & 168 & 0,002 & 0,216 & 0,835 \\
\hline 9 & ,505 & $-1,180$ & 0,323 & 80,741 & $0,748^{*}$ & 0,127 & 0,839 \\
\hline 10 &, 516 &,- 964 & 0,887 & 81,792 & $0,378^{*}$ & 0,152 & 0,837 \\
\hline 11 &,- 698 &,- 720 & $-1,152$ & 84,186 & $0,253^{*}$ & 0,149 & 0,837 \\
\hline 12 &,- 884 &,- 369 & $-0,777$ & 87,648 & $0,439 *$ & 0,151 & 0,837 \\
\hline 13 &,- 009 & $-1,392$ & $-4,421$ & 168 &, 000 & 0,539 & 0,823 \\
\hline 14 &,- 551 & $-1,044$ & $-5,852$ & 168 &, 000 & 0,525 & 0,824 \\
\hline 15 &,- 209 & $-1,173$ & $-8,148$ & 138,623 &, 000 & 0,587 & 0,821 \\
\hline 16 &,- 529 &,- 931 & $-3,119$ & 168 & 0,002 & 0,497 & 0,825 \\
\hline 17 &,- 191 & $-1,083$ & $-5,574$ & 168 &, 000 & 0,554 & 0,823 \\
\hline 18 &,- 413 & $-1,219$ & $-2,614$ & 85,505 & 0,011 & 0,222 & 0,835 \\
\hline 19 &,- 726 &,- 700 & $-6,996$ & 168 &, 000 & 0,546 & 0,823 \\
\hline 20 & $-1,192$ & ,498 & $-3,778$ & 81,471 &, 000 & 0,324 & 0,831 \\
\hline 21 & $-1,357$ &, 728 & $-5,735$ & 83,81 & ,000 & 0,506 & 0,825 \\
\hline 22 & $-1,103$ & 013 & $-7,142$ & 168 & ,000 & 0,532 & 0,824 \\
\hline 23 &,- 880 &,- 394 & $-3,735$ & 81,899 &, 000 & 0,414 & 0,828 \\
\hline 24 &,- 132 & $-1,401$ & $-3,882$ & 168 &, 000 & 0,349 & 0,831 \\
\hline 25 &,- 539 & $-1,087$ & $-1,575$ & 168 & $0,117^{*}$ & 0,288 & 0,833 \\
\hline 26 &,- 198 & $-1,457$ & $-1,32$ & 168 & $0,189^{*}$ & 0,199 & 0,837 \\
\hline 27 &,- 563 &,- 996 & $-5,075$ & 168 & ,000 & 0,475 & 0,826 \\
\hline
\end{tabular}

*Nivel de significación mayor a 0,05 


\section{Análisis Factorial Exploratorio}

Para reducir la dimensionalidad de los datos se empleó el Análisis Factorial Exploratorio, los resultados obtenidos mediante la prueba Kaiser Meyer Olkin (KMO) la adecuación de muestreo fue de $0,84(\mathrm{gl} 351 ; \mathrm{p}<0)$, varianza explicada $39,88 \%$. De la matriz de factores rotados se identificaron y eliminaron los reactivos que cumplían con los siguientes criterios: carga factorial menor a 0,40 , que correlacionaran con más de un factor, reactivos que tuvieran una carga factorial negativa.

El resultado llevo a la depuración de seis reactivos; arrojó una matriz de cuatro factores: Activación, Evitación, Rumiación y Deterioro social, con un total de 21 reactivos (tabla 4), alfa de Cronbach: 0,81 .

Tabla 4. Matriz de factores rotados

\begin{tabular}{|c|c|c|c|c|}
\hline & \multicolumn{4}{|c|}{ Factor } \\
\hline & $\begin{array}{c}1 \\
\text { Activación }\end{array}$ & $\begin{array}{c}2 \\
\text { Rumiación }\end{array}$ & $\begin{array}{c}3 \\
\text { Deterioro } \\
\text { Social }\end{array}$ & $\begin{array}{c}4 \\
\text { Evitación }\end{array}$ \\
\hline $\begin{array}{l}\text { 5, Tomé buenas decisiones respecto a qué tipo } \\
\text { de situaciones y/o actividades hacer. }\end{array}$ & 0,712 & & & \\
\hline $\begin{array}{l}\text { 3. Estuve satisfecho con la cantidad y tipo de } \\
\text { cosas que hice. }\end{array}$ & 0,702 & & & \\
\hline 4. Realicé muchas actividades. & 0,636 & & & \\
\hline $\begin{array}{l}\text { 7. Estuve activo y cumplí los objetivos que me } \\
\text { propuse. }\end{array}$ & 0,612 & & & \\
\hline $\begin{array}{l}\text { 12. Hice algo que fue difícil de lograr pero } \\
\text { valió la pena. }\end{array}$ & 0,591 & & & \\
\hline $\begin{array}{l}\text { 11. Hice cosas aunque fueran difíciles porque } \\
\text { son parte de mis metas a largo plazo. }\end{array}$ & 0,549 & & & \\
\hline 25. Organice mis actividades del día & 0,506 & & & \\
\hline $\begin{array}{l}\text { 17. Pasé tiempo pensando en errores que he } \\
\text { cometido y otras cosas malas en mi historia. }\end{array}$ & & 0,789 & & \\
\hline 15. Pasé tiempo pensando en mi pasado. & & 0,787 & & \\
\hline $\begin{array}{l}\text { 16. Pasé tiempo pensando en personas que me } \\
\text { han lastimado. }\end{array}$ & & 0,733 & & \\
\hline $\begin{array}{l}\text { 13. Pasé mucho tiempo pensando una y otra } \\
\text { vez en mis problemas. }\end{array}$ & & 0,593 & & \\
\hline $\begin{array}{l}\text { 14. Seguí intentando pensar en formas de } \\
\text { resolver un problema. pero nunca intenté } \\
\text { alguna de las soluciones. }\end{array}$ & & 0,404 & & \\
\hline 22. Hice cosas para alejarme de otras personas. & & & 0,633 & \\
\hline 21. Alejé a las personas con mi negatividad. & & & 0,616 & \\
\hline $\begin{array}{l}\text { 19. Estuve alejado y callado. incluso con } \\
\text { personas a las que conozco bien. }\end{array}$ & & & 0,558 & \\
\hline $\begin{array}{l}\text { 1. Me quedé en la cama por mucho tiempo } \\
\text { aunque tenía cosas que hacer y no tenía algún } \\
\text { malestar físico. }\end{array}$ & & & 0,458 & \\
\hline
\end{tabular}




\begin{tabular}{|c|c|c|c|c|}
\hline & \multicolumn{4}{|c|}{ Factor } \\
\hline & $\begin{array}{c}1 \\
\text { Activación }\end{array}$ & $\stackrel{2}{2}$ Rumiación & $\begin{array}{c}3 \\
\text { Deterioro } \\
\text { Social } \\
\end{array}$ & $\begin{array}{l}4 \\
\text { Evitación }\end{array}$ \\
\hline $\begin{array}{l}\text { 23. Me tomé un tiempo libre del trabajo/ } \\
\text { escuela/quehaceres/responsabilidades } \\
\text { simplemente porque no tuve ganas de hacerlo. }\end{array}$ & & & 0,455 & \\
\hline $\begin{array}{l}\text { 27. Empecé a sentirme mal cuando otros } \\
\text { a mí alrededor expresaron sentimientos o } \\
\text { experiencias negativas. }\end{array}$ & & & 0,406 & \\
\hline 10. Traté de no pensar en ciertas cosas. & & & & 0,782 \\
\hline $\begin{array}{l}\text { 9. Hice cosas para evitar sentir tristeza u otras } \\
\text { emociones dolorosas. }\end{array}$ & & & & 0,691 \\
\hline $\begin{array}{l}\text { 26. Sólo me ocupé en actividades que podían } \\
\text { distraerme de sentirme mal. }\end{array}$ & & & & 0,413 \\
\hline Alfa de Cronbach &, 824 & 842 & ,757 & 685 \\
\hline $\begin{array}{l}\text { Método de extracción: Máxima verosimilitud, } \\
\text { Método de rotación: Normalización Varimax co } \\
\text { a, La rotación ha convergido en } 6 \text { iteraciones, }\end{array}$ & Kaiser, & & & \\
\hline
\end{tabular}

\section{Análisis Factorial Confirmatorio}

Después de obtener 21 reactivos agrupados en cuatro factores, se realizó el Análisis Factorial Confirmatorio de Máxima Verosimilitud. Para evaluar el ajuste del modelo se utilizaron: el índice de ajuste comparativo de Bentler CFI, TLI, NFI, RFI, IFI y PNFI; raíz cuadrada media del error de aproximación RMSEA y Chi cuadrada. En todos se logró un buen ajuste: $\mathrm{X}^{2}=1,24 \mathrm{gl}=48 \mathrm{p}=0,118$; $\mathrm{CFI}=0,98$, TLI=0,98, $\mathrm{RMSEA}=0,027, \mathrm{NFI}=0,93, \mathrm{RFI}=0,91, \mathrm{IFI}=0,98, \mathrm{PNFI}=0,68$. La solución final de la escala se muestra en la figura 2.

Figura 2. Modelo de la Escala de Activación Conductual para la Depresión en pacientes con cáncer

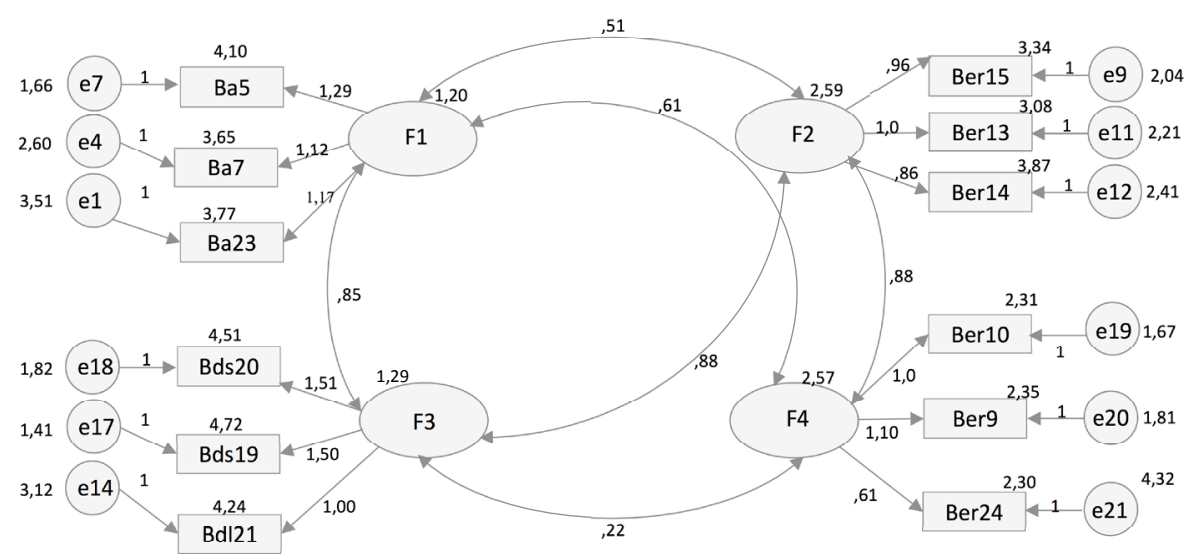


El modelo ajustó después de que se eliminaron nueve reactivos. Como resultado la Escala de Activación Conductual para la Depresión (BADS) (Versión C), quedó con un total de 12 reactivos agrupados en cuatro factores con tres reactivos cada uno: Activación, Deterioro Social, Rumiación y Evitación, en la figura 3 se muestra la lista de reactivos.

Figura 3. Lista de reactivos de la Escala de Activación Conductual para la Depresión para pacientes oncológicos

1. Tomé buenas decisiones respecto a qué actividades hacer.

2. Estuve activo y cumplí los objetivos que me propuse.

3. Hice cosas para evitar sentir tristeza u otras emociones dolorosas.

4. Traté de no pensar en ciertas cosas.

5. Pasé mucho tiempo pensando una y otra vez en mis problemas.

6. Seguí intentando pensar en formas de resolver un problema. pero nunca intenté alguna de las soluciones.

7. Pasé tiempo pensando en mi pasado.

8. Alejé a las personas con mi negatividad.

9. Hice cosas para alejarme de otras personas.

10. Me tomé un tiempo libre del trabajo/escuela/quehaceres/responsabilidades simplemente porque no tuve ganas de hacerlo.

11. Organice mis actividades del día.

12. Sólo me ocupé en actividades que podían distraerme de sentirme mal.

\section{Validez de constructo}

Para determinar la validez de constructo se correlacionó el BADS con los inventarios de ansiedad y depresión de Beck y las escalas de ansiedad y depresión del IMADIS, 122 de los participantes contestaron, además del BADS, los instrumentos de depresión y 107 los correspondientes a ansiedad. El instrumento también se correlacionó con el ECOG, para identificar si el deterioro funcional causado por la enfermedad correlaciona con el nivel de activación conductual de los participantes. El coeficiente de correlación $r$ de Pearson entre BADS y las otras escalas se muestra en la tabla 5.

Tabla 5. Correlación r de Pearson

\begin{tabular}{cc}
\hline Escala & BADS \\
\hline Beck Depresión &,$- 633^{* *}$ \\
IMADIS Depresión &,$- 573^{* *}$ \\
Beck Ansiedad &,$- 517^{* *}$ \\
IMADIS Ansiedad &,$- 482^{* *}$ \\
ECOG &,- 091 \\
\hline$* *$ La correlación es significativa al nivel 0,01 \\
\hline
\end{tabular}




\section{Calificación y normas de BADS}

La escala tiene 7 opciones de respuesta que van de 0 a 6 , los reactivos $3,4,5,6,7,8,9,10$ y 12 se califican de forma inversa, para obtener el puntaje total se suma el valor de cada reactivo de acuerdo a la respuesta del participante. Se obtuvieron las normas de calificación de la adaptación al español de BADS para pacientes oncológicos, el puntaje total se compara con el puntaje crudo, para identificar el nivel de activación conductual (tabla 6).

Tabla 6. Normas BADS al castellano para pacientes oncológicos

\begin{tabular}{cc}
\hline Nivel Activación & Puntaje crudo \\
\hline Poco & $0-31$ \\
Algo & $32-43$ \\
Bastante & $44-55$ \\
Mucho & $56-72$ \\
\hline
\end{tabular}

\section{Discusión y Conclusiones}

Los resultados de este estudio corresponden con los obtenidos en adaptaciones al castellano previas ${ }^{(16,17)}$ y con la escala original ${ }^{(15)}$ la cual tiene propiedades psicométricas adecuadas y también está formada por cuatro factores: activación, evitación/rumiación, deterioro laboral/académico y deterioro social. Sin embargo los factores de la escala BADS para pacientes oncológicos mexicanos son: activación, deterioro social, rumiación y evitación.

El que el factor deterioro académico/laboral, haya sido eliminado en la adaptación para pacientes oncológicos puede deberse a que la mayoría de los participantes eran mujeres cuya ocupación es ama de casa $(58.4 \%)$, lo cual al no ser un trabajo remunerado, pocas veces se considera como empleo, así mismo el 4,7\% de los participantes se encontraban desempleados en el momento de la aplicación y el 10 $\%$ se dedicaba al comercio informal, el cual algunas veces tampoco es visto como un empleo.

Otra diferencia entre la escala $\mathrm{BADS}^{(15)}$ y la adaptación para pacientes oncológicos mexicanos, es que mientras en la primera los reactivos correspondientes a rumiación y evitación se agrupan en un solo factor, en la segunda se agruparon en factores independientes; esto coincide con las áreas de interés a partir de las cuales se desarrolló el instrumento original (dormir y quedarse en cama, actividad, evitación, rumiación, problemas sociales, problemas laborales y académicos, habilidades de afrontamiento, conducta dependiente del estado de ánimo, conciencia e identificación de problemas) y con la hipótesis de los autores de que estos reactivos se agruparían en factores separados.

Al correlacionar la Escala de Activación Conductual para la Depresión para pacientes oncológicos mexicanos con escalas que miden otros constructos relacionados como ansiedad y depresión ${ }^{(25-27)}$, se obtuvo una correlación negativa 
media con ambos constructos; esto se acerca a lo que se esperaba cuando se revisó que el constructo de activación conductual que proporciona la BADS fuera el mismo que se ha considerado en esta investigación y por lo tanto que el nivel de activación conductual reportado por la escala tuviera una correlación significativa negativa fuerte con la depresión y una correlación significativa negativa considerable con el nivel de ansiedad. Por lo tanto esta adaptación de la BADS tiene validez de constructo.

Así mismo cuando se correlacionó la BADS para pacientes con oncológicos con la $\mathrm{ECOG}^{(21)}$ se encontró que no hay correlación significativa entre las escalas. Es decir el nivel de activación conductual no se asocia con el nivel de deterioro funcional causado por la enfermedad, pero si con variables como la ansiedad y la depresión.

Si bien los datos obtenidos en este estudio muestran que la Escala de Activación Conductual para la Depresión para pacientes oncológicos mexicanos, tiene propiedades psicométricas aceptables, se debe tomar en cuenta que la muestra estuvo conformada por pacientes de un solo hospital de la Ciudad de México, en su mayoría por mujeres con cáncer de mama o ginecológico por lo tanto es importante que se realicen más investigaciones en las que se aumente el tamaño de la muestra y la variabilidad de la misma, al incluir a más pacientes con otros diagnósticos oncológicos y que sean atendidos en otros hospitales.

\section{Agradecimientos}

Al Consejo Nacional de Ciencia y Tecnología (CONACYT) por el financiamiento para realizar este estudio, número de beca 449184, número de becario 262337.

\section{Referencias bibliográficas}

1. National Comprehensive Cancer Netwok NCCN Clinical Practice Guidelines in Oncology (NCCN Guidelines). 2018. [Acceso 26 agosto 2018] Disponible en: https:// www.nccn.org/store/login/login.aspx?ReturnURL=https://www.nccn.org/professionals/ physician_gls/pdf/distress.pdf

2. Maté J, Hollenstein M F, Gil FL. Insomnio, ansiedad y depresión en el paciente oncológico. Psicooncología 2004; 1 (2-3): 211-30.

3. Cheung YT, Lee HH-L, Chan A. Exploring clinical determinants and anxiety symptom domains among Asian breast cancer patients. Support Care Cancer 2013;21:2185-94. doi: 10.1007/s00520-013-1769-8.

4. Hoon LS, Chi Sally CW, Hong-Gu H. Effect of psychosocial interventions on outcomes of patients with colorectal cancer: A review of the literature. Eur J Oncol Nurs 2013;17:88391. doi: 10.1016/j.ejon.2013.05.001.

5. Andersen BL, DeRubeis RJ, Berman BS, Gruman J, Champion VL, Massie MJ, et al. Screening, assessment, and care of anxiety and depressive symptoms in adults with cancer: an American Society of Clinical Oncology guideline adaptation. J Clin Oncol 2014; 32:1605-1619. doi: 10.1200/JCO.2013.52.4611

6. Kanter JW, Busch AM, Rusch LC. Activación conductual refuerzos positivos ante la depresión. 1st ed. Madrid: Psicología Alianza Editorial, 2009. 
7. Hopko DR, Armento MEA, Robertson SMC, Ryba MM, Carvalho JP, Colman LK, et al. Brief behavioral activation and problem-solving therapy for depressed breast cancer patients: Randomized trial. J Consult Clin Psychol 2011; 79:834-49. doi:10.1037/ a0025450

8. Ryba MM, Lejuez CW, Hopko DR. Behavioral Activation for Depressed Breast Cancer Patients: The impact of therapeutic compliance and quantity of activities completed on symptom reduction. J Consult Clin Psychol. 2014; 82:325-35. doi: 10.1037/a0035363

9. Fernández RC, Villoria FE, Fernández GP, González FS. Efectos de la activación conductual en la calidad de vida y estado emocional de los pacientes con cáncer de pulmón. Psicooncología 2014; 11:199-215. doi:10.5209/rev_PSIC.2014.v11.n2-3.47383.

10. Hopko DR, Funderburk JS, Shorey RC, McIndoo CC, Ryba MM, File AA, et al. Behavioral activation and problem-solving therapy for depressed breast cancer patients: preliminary support for decreased suicidal ideation. Behav Modif 2013; 37:747-67. doi: $10.1177 / 0145445513501512$

11. Hopko DR, Armento ME, Robertson SM, Ryba MM, Carvalho JP, Colman LK, et al. Brief behavioral activation and problem-solving therapy for depressed breast cancer patients: Randomized trial. J Consult Clin Psychol 2011; 79:834-49. doi: 10.1037/a0025450

12. Fernández C, Villoria E, Amigo I, Padierna C, Gracia J, Fernández R, et al. Terapia de activación conductual en pacientes con cáncer. Ana Psicol 2011; 27:278-91.

13. Hopko DR, Bell JL, Armento ME, Hunt MK, Lejuez CW. Behavior therapy for depressed cancer patients in primary care. Psychotherapy 2005; 42:236-43. doi: 10.1037/00333204.42.2.236

14. Armento ME, Hopko DR. The Environmental Reward Observation Scale (EROS): Development, Validity, and Reliability. Behav Ther 2007; 38:107-19. doi: 10.1016/j. beth.2006.05.003.

15. Kanter J, Mulick P, Busch A, Berlin K, Martel C. The Behavioral Activation for Depression Scale (BADS): Psychometric properties and factor structure. J Psychopathol Behav Assess 2007; 29:191-202. doi: 10.1007/s10862-006-9038-5.

16. Barraca J, Pérez M, Lozano J. Avoidance and activation as keys to depression: adaptation of the Behavioral Activation for Depression Scale in a Spanish sample. Span J Psychol 2011; 14: 998-1009. doi: 10.5209/rev_SJOP.2011.v14.n2.45.

17. Acosta QCO, Vales GJJ, Serrano EDM, Echeverría CS B, García FR. (2017). Confiabilidad y validez de la Escala de Activación Conductual para la Depresión en adultos mayores mexicanos [en prensa].

18. Streiner D, Geoffrey N, Cairney J. Health Measurement Scales. A practical guide to their development and use. 5th ed. Great Britain: Oxford University Press, 2015.

19. Montero I, León O. Clasificación y descripción de las metodologías de investigación en Psicología. Int J Clin Health Psychol 2002; 2:503-508. [Acceso 20 de diciembre de 2019] Disponible en: http://portal.amelica.org/ameli/jatsRepo/33720308

20. Osterlind, S. J. Constructing Test Items. 2nd ed. United Kingdom: Kluwer Academic Publishers, 1989.

21. Oken MM, Creech RH, Tormey DC, Horton J, Davis TE, McFadden ET, et al. Toxicity and response criteria of the Eastern Cooperative Oncology Group. Am J Clin Oncol 1982;5:649-55.

22. Carretero-Dios H, Pérez C. Normas para el desarrollo y revisión de estudios instrumentales. Int J Clin Health Psychol 2005; 5:521-51. [Acceso 20 de diciembre de 2019]. Disponible en: http://www.aepc.es/ijchp/articulos_pdf/ijchp-158.pdf. 
23. Carretero-Dios, H. \& Pérez C. Normas para el desarrollo y revisión de estudios instrumentales: consideraciones sobre la selección de test en la investigación psicológica. Int J Clin Health Psychol 2007; 7:863-82. [Acceso 20 de diciembre de 2019]. Disponible en: http://aepc.es/ijchp/NDREI07_es.pdf

24. Martínez-Arias R. Psicometría: teoría de los test psicológicos y educativos. 1st ed. Madrid: Síntesis, 1995.

25. Jurado CS. Inventario Mexicano de Ansiedad, Depresión e Ideación Suicida (IMADIS). México: Facultad de Psicología UNAM; 2017.

26. Robles R, Varela R, Jurado CS, Páez F. Versión Mexicana del Inventario de Ansiedad de Beck: Propiedades psicométricas. Rev Mex Psicol 2001;2:211-8. [Acceso 20 de diciembre de 2019]. Disponible en: https://biblat.unam.mx/es/revista/revista-mexicana-depsicologia/articulo/version-mexicana-del-inventario-de-ansiedad-de-beck-propiedadespsicometricas.

27. Jurado S, Villegas E, Mendez L, Rodríguez F, Loperena V, Varela R. La estandarización del IDB para residentes de la Ciudad de México. Salud Mental 1998; 21:26-31. 
